\title{
Gadolinium retention after administration of contrast agents based on linear chelators and the recommendations of the European Medicines Agency
}

\author{
Ilona A. Dekkers ${ }^{1} \cdot$ Rick Roos $^{1}$ • Aart J. van der Molen ${ }^{1}$ \\ Received: 24 April 2017 /Revised: 19 August 2017 / Accepted: 7 September 2017 / Published online: 23 October 2017 \\ (C) European Society of Radiology 2017
}

\begin{abstract}
The Pharmacovigilance Risk Assessment Committee (PRAC) of the European Medicines Agency (EMA) earlier this year recommended to suspend some marketing authorisations for Gadolinium Containing Contrast Agents (GCCAs) based on linear chelators due to the potential risk of gadolinium retention in the human body. These recommendations have recently been re-evaluated by EMA's Committee for Medicinal Products for Human Use (CHMP), and confirmed the final opinion of the European Medicines Agency. This editorial provides an overview of the available GCCAs and summarises the recent evidence of gadolinium retention. Moreover, a critical appraisal of the strengths and limitations of the scientific evidence currently available on gadolinium retention is given.

Key points

- EMA recommended suspension of some EU marketing authorisations of four linear GCCAs.

- Brain MRI findings indicating gadolinium retention have been confirmed by mass spectrometry.

- Current scientific evidence for gadolinium retention has several methodological limitations.

- No clear clinical evidence exists indicating that gadolinium retention causes neurotoxicity.

- Long-term safety of GCCAs, however, remains unclear.
\end{abstract}

Ilona A. Dekkers

i.a.dekkers@lumc.nl

1 Department of Radiology, Leiden University Medical Centre, C-2S, Albinusdreef 2, NL-2333, ZA Leiden, The Netherlands
Keywords Contrast media - Magnetic resonance imaging · Gadolinium · Chemical safety $\cdot$ Consumer product safety

$\begin{array}{ll}\begin{array}{ll}\text { Abbreviations } \\ \text { CHMP }\end{array} & \begin{array}{l}\text { Committee for Medicinal Products } \\ \text { for Human Use }\end{array} \\ \text { E-GREC } & \begin{array}{l}\text { European Gadolinium Retention } \\ \text { Evaluation Consortium }\end{array} \\ \text { EMA } & \begin{array}{l}\text { European Medicines Agency } \\ \text { FDA }\end{array} \\ \text { USCAs Food and Drug Administration } \\ \text { Gd } & \begin{array}{l}\text { Gadolinium-containing contrast agents } \\ \text { Gadolinium }\end{array} \\ \text { NSF } & \text { Nephrogenic systemic fibrosis } \\ \text { PRAC } & \text { Pharmacovigilance Risk Assessment } \\ & \text { Committee }\end{array}$

\section{Introduction}

The Pharmacovigilance Risk Assessment Committee (PRAC) of the European Medicines Agency (EMA) earlier this year recommended suspending some marketing authorisations for gadolinium-containing contrast agents (GCCAs) based on linear chelators due to the potential risk of gadolinium retention in the human body [1]. The recommendations made by the PRAC were based on the evaluation of recent research findings indicating gadolinium retention in the brain, which can be measured as increased signal intensities on T1-weighted sequences of unenhanced MRI scans. The PRAC has re-evaluated its recommendations based on questions by the marketing authorisation holders, which has subsequently been confirmed by the Committee for Medicinal Products for Human Use (CHMP). 
Table 1 Current arsenal of linear and macrocyclic gadolinium-based contrast agents (GBCAs). Classification of available GBCAs into four different groups based on the type of ligand (linear or macrocyclic) and charge (ionic or non-ionic), with corresponding brand name and manufacturer

\begin{tabular}{ll}
\hline Linear & Macrocyclic \\
\hline Ionic & Ionic \\
Magnevist (gadopentetate dimeglumine - Bayer) & Dotarem (gadoterate meglumine - Guerbet) \\
Magnevision (gadopentetate dimeglumine - B.E. Imaging) & Clariscan (gadoterate meglumine - GE Healthcare) \\
& Dotagraf (gadoterate meglumine - Bayer) \\
& Dotagita (gadoterate meglumine - Agfa) \\
& Cyclolux (gadoterate meglumine - Sanochemia)
\end{tabular}

MultiHance (gadobenate dimeglumine - Bracco)

Primovist (gadoxetate disodium - Bayer)

Non-ionic

Non-ionic

Omniscan (gadodiamide - GE Healthcare)

ProHance (gadoteridol - Bracco)

OptiMARK (gadoversetamide - Guerbet)

Gadovist (gadobutrol - Bayer)

The CHMP is a regulatory organ within the EMA with a central role in the authorisation of medicines inside the European Union (EU). On 21 July 2017, the EMA published its final opinion to suspend and/or restrict some of the marketing authorisations of four GCCAs based on linear chelators from the European market [2]. These four agents based on linear chelators are gadobenate dimeglumine (MultiHance), gadodiamide (Omniscan), gadopentetate dimeglumine (Magnevist) and gadoversetamide (OptiMark). This is not the first time that linear gadolinium agents are under investigation by the EMA. In 2007, exposure to high doses of gadolinium were for the first time linked to the development of nephrogenic systemic fibrosis (NSF) in patients with severe renal insufficiency. In this period, the EMA gave a serious warning (e.g. 'Black Box' warning) regarding the relationship between NSF and linear chelators; however, the scientific evidence at that time was considered insufficient to proceed with a ban from the European market. However, how strong is the scientific evidence for the current recommendations and what are the implications for radiology practice in the EU?

\section{Physical chemistry of gadolinium-containing contrast agents (GCCAs)}

GCCAs can be classified into four different groups based on the type of ligand (linear or macrocyclic) and charge (ionic or nonionic; Table 1). Gadolinium is a heavy metal from the lanthanide group with strong paramagnetic properties (shortening of the proton $\mathrm{T} 1$ relaxation time), which is chelated by binding to a strong ligand. In the linear chelates the gadolinium ion is bound to an open-chain ligand, while in the macrocyclic chelates the gadolinium ion is bound inside a cubic chemical structure. These differences in the chemical structure of the ligands explain the difference in thermodynamic and kinetic stability, whereby the non-ionic linear chelates are the least stable and the ionic macrocyclic chelates are the most stable [3]. The free gadolinium ion is mostly hydrated in biological systems, and this $\mathrm{Gd}\left(\mathrm{H}_{2} \mathrm{O}\right)_{8}{ }^{3+}$ ion is toxic because of its chemical similarities to $\mathrm{Ca}^{2+}$, which is an important factor for proper functioning of many processes in the human body such as contraction of the heart muscle and smooth muscle cells, and nerve transmission. $\mathrm{Gd}^{3+}$ can compete with $\mathrm{Ca}^{2+}$ due to its similarity in ion radius, and could thereby disturb physiological processes. In unstable chelates, the gadolinium ion could, partly depending on the local environment (acidic $\mathrm{pH}$ ), become detached from the ligand by transmetallation (exchange with other ions present in the local environment, such as $\mathrm{Fe}^{3+}, \mathrm{Cu}^{2+}$, or $\mathrm{Zn}^{2+}$ ions) and the $\mathrm{Gd}^{3+}$ ion could then precipitate locally as a salt (gadolinium hydroxide, gadolinium carbonate or gadolinium phosphate) or bind to other macromolecules, such as proteins, peptides or metalloenzymes [4].

\section{Evidence for gadolinium retention}

In 2014, Kanda et al. described a positive correlation between previous exposure to GCCAs based on linear chelators and increased signal intensity in basal ganglia on subsequent unenhanced T1-weighted MRI sequences in 35 patients [5]. This increase in signal intensity was not found in the control group, which consisted of patients who underwent multiple MRI scans without the addition of a contrast agent. These findings were soon replicated by multiple independent research groups in different countries. To date, a total of 19 studies have been performed that investigated whether repeated exposure to GCCAs causes increased signal intensity in the brain. Thus far, 15 out of 19 studies found a positive correlation between the number of administrations of a linear chelate and the measured signal intensity in the basal ganglia. At this moment, the association has not been demonstrated for the macrocyclic chelates [6, 7]. The four contrast agents that the EMA has recommended suspending and/or restricting 


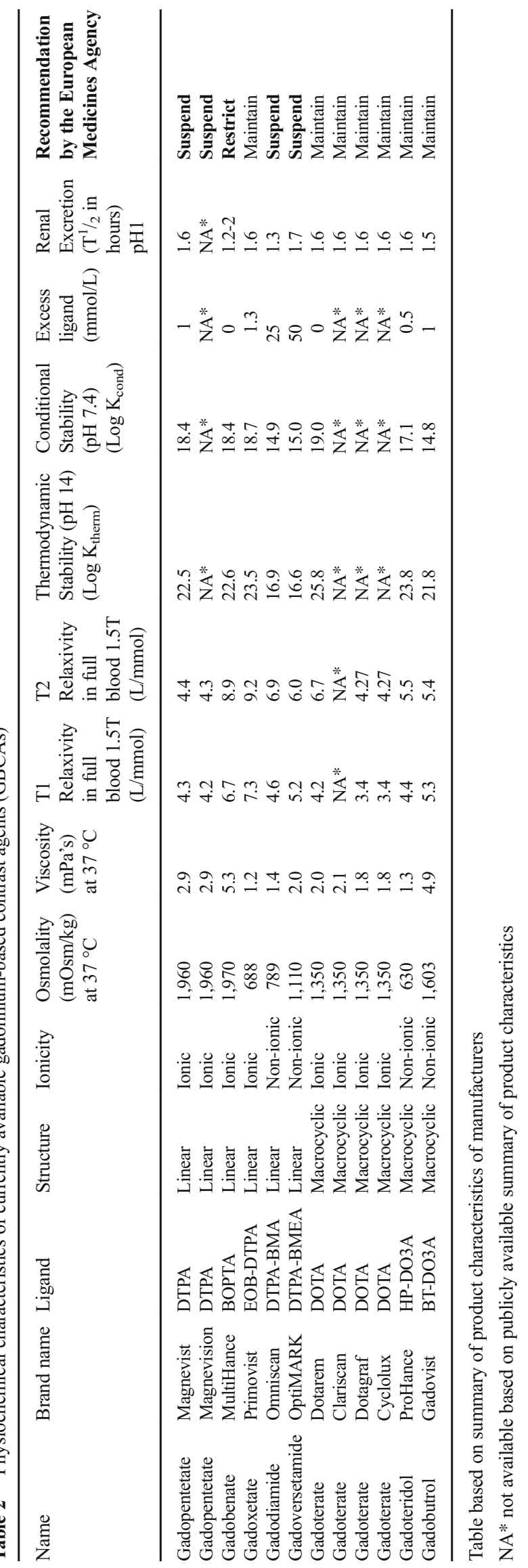

marketing authorisations are all based on linear chelators (see Table 2). Furthermore, small post-mortem studies showed the presence of miniscule amounts of gadolinium in brain tissue in patients who received GCCAs in the past [8-10]. The recent post-mortem study by Murata et al. found that gadolinium deposition in normal brain and bone tissue occurs with both agents based on macrocyclic (only nonionic, i.e. gadobutrol and gadoteridol) and linear chelators in patients with normal renal function [10]. Gadolinium retention in bone and skin had already been described by histology studies [11, 12], and very recent data based on autopsy studies suggest that gadolinium can accumulate in other organs as well, such as the liver and kidney $[13,14]$.

\section{Methodological limitations of current studies}

The studies performed thus far have several important limitations that need to be considered when interpreting these data. All studies have an observational retrospective design, which makes these studies vulnerable to different forms of bias and confounding. The retrospective data collection introduces uncertainty about the number of administered doses and included patients could have received different types of contrast agents rather than solely linear or macrocyclic, which could lead to misclassification bias. Furthermore, only few studies have sufficiently corrected for potential confounders that could distort the association between gadolinium exposure and an increased signal intensity in the brain. Examples of such potential confounders are age, gender and follow-up time. Control groups were also not always included in the analyses and if present were often limited to small sample sizes and comprised different patient populations compared to the exposed group. Besides the above-mentioned methodological drawbacks, there are also significant limitations to the use of increased signal intensity as a primary outcome measure. The increased signal intensity in the brain on MRI scans is not always reliable and comparable due to different field strengths (1.5 Tesla and 3.0 Tesla), and the use of different T1-weighted sequences or sequence parameters. Moreover, the proton T1 relaxivity also depends on the molecular weight of the molecule to which the Gd-ions or Gd-contrast media molecules are attached (either soluble macromolecules or insoluble cell components), the water residence time of the coordinated water molecules, and, for some GCCAs, the presence of albumin [15, 16]. It is therefore unclear to what extent the increased signal intensity in the brain truly reflects higher gadolinium concentrations [17]. Each of the GCCAs possibly has a different level of administered doses before signal changes can be observed on MR images. It could be that for agents based on macrocyclic chelators this level involves such a substantially high number of administrations, which hardly occurs in individual patients and is therefore difficult to investigate. 
Thus far, five post-mortem studies, all using inductively coupled plasma mass spectroscopy, have measured levels of elemental Gd in different brain areas with possibly intact blood-brain barriers [8, 10, 17-19]. Overall, data are limited to 31 patients exposed to either linear or macrocyclic-based GCCAs compared to 28 controls. Only one study $(\mathrm{N}=13)$ has assessed the possible distribution of Gd compounds in brain tissues, and the possible presence of histological changes [18]. To summarise, these studies found that Gd deposition has been observed in all 31 patients exposed to either linear or macrocyclic GCCAs, even after one single exposure, compared to controls. Second, calculation of normalised Gd deposition ratios (Gd deposited in $1 \mathrm{~g}$ of tissue per millimole of GCCA administered) did not show significant differences between linear and macrocyclic GCCAs. In contrast to the imagingbased studies, the post-mortem study of Murata et al. suggests that gadolinium retention is not solely limited to agents based on linear chelators [8]. This suggests that extension of the suspension of marketing authorisations to certain GCCAs based on macrocyclic chelators cannot be completely excluded if more evidence becomes available. However, the current number of patients studied in tissue analysis is too small to draw any conclusions on differences among the various GCCAs tested, and does not allow for differentiation between the various forms of gadolinium (e.g. chelated Gd, Gd-associated macromolecules) as different GCCAs with various cumulative doses and with heterogeneous sampling times have been studied.

The association between gadolinium accumulation and the occurrence of physical symptoms or neurotoxic damage has not been demonstrated yet. Thus far, two studies aimed to assess the possible influence of gadolinium accumulation and clinical symptoms $[20,21]$. The study by Welk et al. involving 99,739 patients with one or more Gd-enhanced MRI and 146,818 control patients with only non-enhanced MRIs did not find a significant association with parkinsonism [20]. A very recent retrospective cohort study involving nearly 20 years of longitudinal data in 23 multiple sclerosis patients and 23 healthy age- and sex-matched controls found a possible association between $\mathrm{Gd}$ exposure and cognitive impairment (verbal fluency) [21]. However, it should be noted that these findings need to be interpreted with caution since retrospective studies are highly susceptible to 'confounding by indication'. Patients requiring contrast administration in general tend to be more ill than patients in whom contrast administration is not needed, which biases the assessment of the effect of contrast media and clinical outcome. To test the association between $\mathrm{Gd}$ and clinical symptoms, one would ideally do a prospective study with clinical symptoms as the study outcome, whereby the patients are randomised for receiving contrast agents or not. However, a prospective study on rare clinical outcomes is impracticable since very large sample sizes are needed and are, furthermore, unethical. Previous animal studies evaluating side effects of lanthanum carbonate, which is a rare metal of the same lanthanide family as gadolinium, have related this agent to reduced learning behaviour in rats at lower concentrations than now found in humans for gadolinium [22]. However, no direct neurotoxicity endpoints were evaluated [23], and adverse effects on learning behaviour in both humans and animals have not yet been described for gadolinium. Nevertheless, caution is needed regarding patient safety and unknown long-term effects. In this view, the advice of the PRAC is understandable and probably a safe decision considering the availability of several different classes of GCCAs (Table 1). Additionally, as radiologists it is advisable to keep in mind that for every diagnostic examination, especially when using contrast agents or radiation, a clear clinical indication should be present, and to keep the contrast agent dose as low as reasonably possible.

\section{Implications}

Following the PRAC's March 2017 recommendation, some of the marketing authorisation holders concerned by this referral procedure have requested a re-examination. Upon receipt of the grounds for their requests, the PRAC has completed a re-examination, which was published on 7 July 2017. An additional assessment was performed by the CHMP, which was unfavourable for the four linear contrast agents regarding quality, safety and efficacy requirements and risk-benefit analysis. The GCCAs that fulfil an important diagnostic need in patients with few alternatives such as the hepatobiliary specific linear agent gadoxetic acid and a formulation of gadopentetic acid used for MR arthrography, were excluded from the PRAC investigation and will thus maintain their marketing authorisation. In line with contrast agents fulfilling specific diagnostic needs, the use of gadobenic acid has been restricted to liver MRI scans. The reactions to the statement in the field have been diverse. For example, the Committee of Contrast Media and Drugs of the American College of Radiology previously noted finding the statement of the PRAC premature and are not in favour of the suspension of marketing authorisations of these agents for the US market based on the current available data [24]. In May 2017 the US Food and Drug Administration (FDA) announced a safety update regarding the evaluation of the risk of Gd accumulation associated with repeated administration of GCCAs. The FDA concluded that although GCCAs may be associated with some Gd retention in the brain and other organs, no adverse health effects from Gd retention have been identified. Previous recommendations of the FDA regarding the use of GCCAs remain thus unchanged [25]. 


\section{International research cooperation}

Since gadolinium retention in the body (in the brain but also in bone, skin and liver) is a rare phenomenon, multiple centres in Europe are collaborating to achieve international cooperation on this topic. The European Gadolinium Retention Evaluation Consortium (E-GREC), founded in 2016, is a collaboration of academic clinical researchers and basic researchers from the contrast agent manufacturers [26]. Additionally, the consortium has close connections to researchers in the USA. EGREC will initially focus on acquiring resources to fund future scientific projects and will focus on guidelines to improve the quality of the preclinical and clinical research on gadolinium retention.

Funding The authors state that this work has not received any funding.

\section{Compliance with ethical standards}

Disclaimer Ilona A. Dekkers is a member of the Dutch Guidelines Committee on the Safe Use of Contrast Agents, Part Two.

Aart J. van der Molen is a member of the Contrast Media Safety Committee of the European Society of Urogenital Radiology and secretary of the European Gadolinium Retention Evaluation Consortium.

Aart J. van der Molen is the chairmen of the Dutch Guidelines Committee on the Safe Use of Contrast Agents, Parts One and Two.

The opinions in this article are personal and they are not the official opinions of the committees.

Guarantor The scientific guarantor of this publication is Aart van der Molen.

Conflict of interest Aart van der Molen declares relationships with the following company:

Guerbet.

Ilona A. Dekkers and Rick Roos declare no relationships with any companies whose products or services may be related to the subject matter of the article.

Statistics and biometry No complex statistical methods were necessary for this paper.

Informed consent Written informed consent was not required for this study because this is an editorial.

Ethical approval Institutional Review Board approval was not required because this is an editorial.

\section{References}

1. (2017) PRAC concludes assessment of gadolinium agents used in body scans and recommends regulatory actions, including suspension for some marketing authorisations EMA/157486/2017. Pharmacovigilance Risk Assessment Committee

2. (2017) EMA's final opinion confirms restrictions on use of linear gadolinium agents in body scans EMA/457616/2017. European Medicines Agency
3. Sherry AD, Caravan P, Lenkinski RE (2009) Primer on gadolinium chemistry. J Magn Reson Imaging 30:1240-1248

4. Idee JM, Port M, Raynal I, Schaefer M, Le Greneur S, Corot C (2006) Clinical and biological consequences of transmetallation induced by contrast agents for magnetic resonance imaging: a review. Fundam Clin Pharmacol 20:563-576

5. Kanda T, Ishii K, Kawaguchi H, Kitajima K, Takenaka D (2014) High signal intensity in the dentate nucleus and globus pallidus on unenhanced T1-weighted MR images: relationship with increasing cumulative dose of a gadolinium-based contrast material. Radiology 270:834-841

6. Radbruch A, Weberling LD, Kieslich PJ et al (2015) Gadolinium retention in the dentate nucleus and globus pallidus is dependent on the class of contrast agent. Radiology 275:783-791

7. Olchowy C, Cebulski K, Lasecki M et al (2017) The presence of the gadolinium-based contrast agent depositions in the brain and symptoms of gadolinium neurotoxicity - A systematic review. PLoS One 12:e171704

8. Kanda T, Fukusato T, Matsuda M et al (2015) Gadolinium-based Contrast Agent Accumulates in the Brain Even in Subjects without Severe Renal Dysfunction: Evaluation of Autopsy Brain Specimens with Inductively Coupled Plasma Mass Spectroscopy. Radiology 276:228-232

9. Kanda T, Oba H, Toyoda K, Kitajima K, Furui S (2016) Brain gadolinium deposition after administration of gadolinium-based contrast agents. Jpn J Radiol 34:3-9

10. Murata N, Gonzalez-Cuyar LF, Murata K et al (2016) Macrocyclic and Other Non-Group 1 Gadolinium Contrast Agents Deposit Low Levels of Gadolinium in Brain and Bone Tissue: Preliminary Results From 9 Patients With Normal Renal Function. Invest Radiol 51:447-453

11. Darrah TH, Prutsman-Pfeiffer JJ, Poreda RJ, Ellen Campbell M, Hauschka PV, Hannigan RE (2009) Incorporation of excess gadolinium into human bone from medical contrast agents. Metallomics $1: 479-488$

12. van der Meij N, Keur I, van Lienden KP, Scheepstra CG, Bos JD (2007) Nephrogenic systemic fibrosis possibly caused by gadolinium-containing contrast agent. Ned Tijdschr Geneeskd 151:2898-2903

13. McDonald J, Jentoft M, Paolini M et al (2017) Deposition of Gadolinium in the Organs, Tissue, and Bone of Patients who Underwent Multiple Gadodiamide-Enhanced MR Exams. Mayo Clinic, Rochester, MN, ASNR O-259

14. McDonald RJ, McDonald JS, Dai D et al (2017) Comparison of gadolinium concentrations within multiple rat organs after intravenous administration of linear versus macrocyclic gadolinium chelates. Radiology:161594

15. Frenzel T, Apte C, Jost G, Schöckel L, Lohrke J, Pietsch H (2017) Quantification and assessment of the chemical form of residual gadolinium in the brain after repeated administration of gadolinium-based contrast agents: comparative study in rats. Invest Radiol 52:396-404

16. Idée J-M, Fretellier N, Robic C, Corot C (2014) The role of gadolinium chelates in the mechanism of nephrogenic systemic fibrosis: a critical update. Critical reviews in toxicology 44:895-913

17. Roberts DR, Welsh CA, LeBel DP 2nd, Davis WC (2017) Distribution map of gadolinium deposition within the cerebellum following GBCA administration. Neurology 88:1206-1208

18. McDonald RJ, McDonald JS, Kallmes DF et al (2015) Intracranial Gadolinium Deposition after Contrast-enhanced MR Imaging. Radiology 275:772-782

19. McDonald JS, McDonald RJ, Jentoft ME et al (2017) Intracranial Gadolinium Deposition Following Gadodiamide-Enhanced Magnetic Resonance Imaging in Pediatric Patients: A CaseControl Study. JAMA Pediatr. https://doi.org/10.1001/ jamapediatrics.2017.0264 
20. Welk B, McArthur E, Morrow SA et al (2016) Association Between Gadolinium Contrast Exposure and the Risk of Parkinsonism. Jama 316:96-98

21. Forslin Y, Shams S, Hashim F et al (2017) Retention of Gadolinium-Based Contrast Agents in Multiple Sclerosis: Retrospective Analysis of an 18-Year Longitudinal Study. AJNR Am J Neuroradiol. https://doi.org/10.3174/ajnr.A5211

22. Feng L, Xiao H, He X et al (2006) Neurotoxicological consequence of long-term exposure to lanthanum. Toxicol Lett 165:112-120
23. Damment SJ, De Broe ME, D'Haese PC, Bramall N, Cox AG, McLeod CW (2007) Incredulous effects of lanthanum? Toxicol Lett 168:186-189

24. (2017) ACR Response to the European PRAC Recommendations. American College of Radiology

25. (2017) FDA identifies no harmful effects to date with brain retention of gadolinium-based contrast agents for MRIs. Food and Drug Administration

26. Quattrocchi CC, van der Molen AJ (2017) Gadolinium Retention in the Body and Brain: Is It Time for an International Joint Research Effort? Radiology 282:12-16 